\section{PC2-mitochondria interactions in PKD}

Interactions between the endoplasmic reticulum (ER) and mitochondria occur at the mitochondria-associated ER membrane (MAM). This region regulates mitochondrial metabolism, in part through the transfer of $\mathrm{Ca}^{2+}$ from the ER to mitochondria. Researchers now demonstrate that polycystin 2 (PC2) modulates proteins at the MAM to regulate $\mathrm{Ca}^{2+}$ signalling and mitochondrial dynamics. "Our results show that $\mathrm{PC} 2$ has profound effects on cell metabolism through a $\mathrm{Ca}^{2+}$-dependent mitochondrial process," says Barbara Ehrlich.

Loss-of-function mutations in the genes that encode the polycystins PC1 and PC2 result in autosomal dominant polycystic kidney disease (ADPKD). Previous research suggested that cyst development is associated with metabolic changes; however, the role of PC2 in this process was unclear. "The fact that $\mathrm{PC} 2$ resides at a location in the ER membrane that enables it to potentially modulate mitochondrial metabolism led us to hypothesize that PC2-regulated changes in $\mathrm{Ca}^{2+}$ signalling might alter mitochondrial function," says Ehrlich. Using cell lines, Ivana Kuo, Allison Brill and colleagues show that knockdown (KD) of PC2 enhances ER-mediated mitochondrial $\mathrm{Ca}^{2+}$ influx. This influx was associated with increased tethering of the ER to mitochondria and increased expression of the mitochondrial tethering protein MFN2. Mitochondria of PC2-KD cells also exhibited increased metabolic activity and reduced movement, indicative of prolonged interaction between mitochondria and the ER.

Similar to PC2-KD cells, cystic renal cells of PC2-knockout mice had increased MFN2 levels and enhanced mitochondrial $\mathrm{Ca}^{2+}$ influx. KD of MFN2 in the kidneys of these mice restored mitochondrial $\mathrm{Ca}^{2+}$ to control levels and reduced proliferation in kidney cysts. Finally, cyst-lining cells from human patients with ADPKD also demonstrated increased MFN2 expression. "We are incredibly excited by our finding that targeting the connections between the ER and mitochondria to reduce $\mathrm{Ca}^{2+}$ transfer decreases cell proliferation in our cystic mouse models," says Ehrlich. "Allison Brill is now investigating how PC2 and its downstream signalling partners could be targeted to modulate mitochondrial function," she adds.

Susan J. Allison

ORIGINAL ARTICLE Kuo, I. Y. et al. Polycystin 2 regulates mitochondrial $\mathrm{Ca}^{2+}$ signaling, bioenergetics, and dynamics through mitofusin 2. Sci. Signal. 12, eaat7397 (2019)

$\Rightarrow$ ACUTE KIDNEY INJURY

\title{
A molecular imaging approach for the early, real-time diagnosis of acute kidney injury
}

The timely diagnosis of acute kidney injury (AKI) is hindered by a lack of approaches to detect early-stage molecular-level changes that underlie renal injury. Now, Kanyi Pu and colleagues describe the development of optical molecular renal probes (MRPs) to enable real-time imaging of three early-stage biomarkers - superoxide anion $\left(\mathrm{O}_{2}^{--}\right)$, $N$-acetyl- $\beta$-D-glucosaminidase (NAG) and caspase-3 - in mouse models of druginduced AKI. "Our smart MRPs specifically interact with kidney biomarkers that are indicative of oxidative stress, lysosomal damage and cellular apoptosis," says $\mathrm{Pu}$. "Subtle changes in these biomarkers occur in the kidneys of living animals before the development of tissue damage, enabling early diagnosis of AKI."

To non-invasively detect molecular-level changes in the kidney in response to injury, $\mathrm{Pu}$ and colleagues developed probes MRP1-3 that emit near-infrared fluorescence signals in the presence of $\mathrm{O}_{2}{ }^{-}$, NAG and caspase-3, respectively. "Our probes undergo renal clearance with nearly $100 \%$ efficiency, meaning that the signals we detect are from the kidneys," explains Pu. Longitudinal imaging of the MRPs following their injection into cisplatin-treated mice demonstrated sequential activation of the probes, indicating the sequential occurrence of oxidative stress, lysosomal damage and cellular apoptosis. "These changes occurred prior to a decrease in glomerular filtration," says $\mathrm{Pu}$. "In addition, urinalysis of the excreted probes detected the onset of cisplatin or gentamicin-induced AKI 24-72 hours earlier than assays of NGAL, cystatin C or $\beta_{2}$-microglobulin."

The researchers plan to test their probes in clinical urine samples. "Our work not only permits mechanistic investigation of nephrotoxicity and high-throughput drug screening in living animals, but also provides a convenient and potentially better way to monitor the kidney status of patients in real time," Pu adds.

Susan J. Allison

ORIGINAL ARTICLE Huang, J. et al. Molecular optical imaging probes for early diagnosis of drug-induced acute kidney injury. Nat.Mater. https://doi.org/10.1038/s41563-019-0378-4 (2019)

\section{GENETICS}

\section{A million genotypes to find CKD-linked loci}

Genome-wide association studies (GWAS) are often used to study complex disorders such as chronic kidney disease (CKD), which represents a substantial global health burden. "Larger sample sizes, better statistical methods and new publicly available omics data sets enabled us to detect novel lower frequency variants associated with CKD, and to map causal genes," report Anna Köttgen, Cristian Pattaro and colleagues.

In a trans-ancestry meta-analysis of data from $>700,000$ individuals, the researchers first identified genome-wide significant loci associated with creatinine-based estimated glomerular filtration rate (eGFR). "These loc explain nearly $20 \%$ of eGFR genetic heritability, which doubles previous estimates," remarks Pattaro. Another GWAS cohort of nearly 300,000 individuals enabled the replication of 264 single-nucleotide polymorphisms (SNPs), of which 147 were significantly associated with blood urea nitrogen (BUN) and prioritized for analysis as they were more likely to be directly linked to renal function, not just creatinine metabolism. In fact, a genetic risk score based on these 147 SNPs was associated with higher odds ratios of renal disease in a cohort of nearly 0.5 million individuals. "These results support the clinical and epidemiological relevance of our findings," remarks Köttgen.

The researchers also performed colocalization analyses of transcripts mapped to eGFR-associated loci with gene expression data from 46 tissues, including micro-dissected renal tubules and glomeruli. "This is an unbiased way to highlight the most likely causal genes and tissues," explains Pattaro. "Fine-mapping analysis also allowed us to narrow down GWAS association signals to single causal variants or genes, and we identified missense variants in 11 genes."

"We created a large list of candidate genes for translational research and all our results are publicly available," notes Köttgen. "This is perhaps one of the most important features of this kind of large-scale effort: the generation and sharing of a resource that can be used for many additional projects."

Monica Wang

ORIGINAL ARTICLE Wuttke, M. et al. A catalog of genetic loci associated with kidney function from analyses of a million individuals. Nat. Genet. 51, 957-972 (2019) 